\title{
Factores que incidieron en el emprendimiento rural en Extremadura (España) durante el período 2003 - 2012 1
}

\section{Factors that influenced rural entrepreneurship in Extremadura (Spain) during 2003 - 2012 period}

\begin{abstract}
Resumen
El emprendimiento puede ayudar a solucionar algunos problemas de la sociedad actual; en las áreas rurales podría influenciar positivamente la economía, generar empleo, frenar la despoblación y dinamizar estos espacios. Sin embargo, el entorno donde se lleve a cabo puede condicionarlo. El objetivo de este trabajo fue identificar y analizar las condiciones del entorno emprendedor (EFCS) que impulsaron o dificultaron el emprendimiento rural en Extremadura durante el período 2003 - 2012. La investigación realizada fue descriptiva; la información utilizada provino de la encuesta a expertos en el entorno para emprender del proyecto Global Entrepreneurship Monitor (GEM) Extremadura. Los resultados permiten observar que el acceso a la infraestructura física, el apoyo a la mujer y la innovación, fueron las condiciones que impulsaron el emprendimiento; por su parte, la educación, las habilidades y el apoyo financiero, al parecer obstaculizaron la aparición de nuevas empresas en Extremadura.
\end{abstract}

\section{Palabras clave}

Factores del entorno, emprendimiento, Extremadura, rural, actividad emprendedora.

\section{Códigos de clasificación JEL: L26, 018, 031, 032, R51}

\begin{abstract}
Entrepreneurship can help to solve some problems of current's society; in rural areas it could influence economy positively, generate employment, curb depopulation and stimulate these spaces. However, the environment in which it will take place can condition it. The aim of this paper was to identify and analyze the Entrepreneurial Framework Conditions (EFCs) that drove or hinder rural entrepreneurship in Extremadura during 2003 - 2012 period. The research was descriptive; the information used came from of experts in the environment to undertake survey of the Global Entrepreneurship Monitor (GEM) Extremadura Project. The results allow us to observe that access to physical infrastructure, women's support and innovation, were the conditions that drove the entrepreneurship; in the other side education, skills and financial support, apparently hindered the new businesses creation in Extremadura.
\end{abstract}

\section{Keywords}

Framework entrepreneurial conditions, entrepreneurship, Extremadura, rural, entrepreneurial activity.

\section{Artículo de investigación.}

2 Universidad Santo Tomás Seccional Bucaramanga. Correo electrónico: leidykgarcia@gmail.com. Código ORCID: 0000-0003-3369-7417

$3 \quad$ Universidad de Extremadura (España).Correo electrónico: cdiaz@unex.es 


\section{Introducción}

Muchos economistas afirman que la actividad emprendedora es vital para el crecimiento de las economías (Stel, Carree y Thurik, 2005). De igual forma, parece ser que la creación de nuevas empresas es importante, pues genera nuevos empleos, apoya el desarrollo de los países o regiones, ayuda a mejorar la competitividad de los territorios, incide positivamente en la innovación y en la construcción de un desarrollo sostenible, $\mathrm{y}$, fomenta el bienestar humano y la reducción de la pobreza (Acs y Amorós, 2008; Amorós y Poblete, 2013; Amorós y Bosma, 2014; Aspen Network of Development Entrepreneurs, 2013; Coduras, Urbano, Rojas y Martínez, 2008; Poblete y Amorós, 2013; Porter, 1991; Sánchez, Postigo, Díaz y Hernández, 2012; World Economic Forum - WEF, 2009), por tanto, se podría inferir que la creación de empresas proporciona un buen camino para alcanzar los desafíos que enfrenta la sociedad actual.

Algunas teorías socioculturales e institucionales que estudian el fenómeno de la creación de empresas sugieren que estas se vinculan a los factores del entorno en que se crean y que estos influyen en el comportamiento de los emprendedores (Díaz, Pulido y Hernández, 2005). Por una parte, parece ser que el entorno económico afecta a la dinámica de la actividad empresarial (Acs y Amorós, 2008); de igual forma, el marco institucional condiciona el nacimiento y la evolución de las empresas, entendiendo que las instituciones corresponden tanto a factores formales (políticas gubernamentales, mecanismos de fomento de la actividad empresarial, etc.), como informales (actitudes de la sociedad hacia la creación de empresas), y ambos condicionan la creación de empresas (Díaz et al., 2005). A su vez, los factores socioculturales como la religión, la familia, el entorno empresarial, la sociedad, la cultura, entre otros, también pueden influir en la actividad emprendedora (Díaz et al., 2005). En este sentido, es importante conocer los factores que integran el ecosistema en el que se da el emprendimiento y su comportamiento, puesto que brindará información para proponer estrategias que lo estimulen y, por tanto, influyan positivamente en el desarrollo de las regiones (Hernández y González, 2015; WEF, 2009).

Las áreas rurales de Extremadura, al parecer, enfrentan a una serie de problemas como la falta de población y su progresivo envejecimiento, el éxodo rural, la escasa diversificación económica, la alta tasa de desempleo, la masculinización, los bajos niveles de natalidad, la falta de algunos servicios, entre otros. Estos problemas requieren alternativas de solución, una de estas puede ser diversificar las actividades económicas que allí se realizan e impulsar el tejido empresarial, con el fin de mejorar las condiciones y el desarrollo del territorio e incidir en la mejora de la calidad de vida de la población (Gobierno de Extremadura, 2013); en este sentido, fomentar el emprendimiento en el medio rural de la comunidad autónoma (CA) es una buena alternativa para enfrentar los problemas descritos. 


\section{Planteamiento del problema y justificación}

Parece ser que las realidades económicas están ocurriendo más rápidamente que las políticas. La pasada crisis financiera del 2008 afectó negativamente al progreso económico, social y la creación de empleo que se había registrado en Europa durante la última década, provocando un fuerte impacto para millones de ciudadanos y reflejando algunas carencias en la economía, que entrevén un momento de transformación (Comisión Europea, 2012). Ante esto, uno de los efectos de la crisis financiera ha sido la revitalización de la importancia del espíritu emprendedor y la iniciativa empresarial, como mecanismo para mejorar las economías y el bienestar de los países (WEF, 2009).

Si bien, el crecimiento económico, la innovación de las empresas, la creación de empleo, la despoblación de las áreas rurales son problemas que han venido afectando a la sociedad y, por ende, a los gobiernos en las últimas décadas, en muchos territorios. El emprendimiento parece haberse manifestado como una de las posibles soluciones, puesto que incide en el desarrollo y crecimiento económico de los países, impulsa la innovación, la generación de nuevas empresas, la creación de empleo abre nuevos mercados, favorece nuevas competencias y capacidades, garantiza el pago de impuestos futuros, ayuda a estimular la actividad económica y la competitividad. Además, fomenta la cohesión económica y social en las áreas rurales e involucra la participación de todos los grupos de la sociedad, incluidas las mujeres, diferentes grupos etarios y educativos, y las minorías desfavorecidas (Amorós y Poblete, 2013; Comisión Europea, 2003; Díaz, 2003; Sánchez et al., 2012).

Esta situación ha propiciado el impulso de diversas estrategias para favorecer la actividad emprendedora en los territorios por parte de las administraciones públicas (Coduras et al., 2008; Comisión Europea, 2012a; Díaz, Hernández, Sánchez y Postigo, 2010; Hernández y González, 2015) y, por tanto, que la iniciativa emprendedora esté presente en los procesos de desarrollo territorial y del tejido productivo de la sociedad moderna (Ministerio de Industria, Turismo y Comercio - Observatorio PYME, 2010).

Uno de los principales problemas que afecta a la economía española, está relacionado con la alta tasa de desempleo existente, la cual en el 2015 se ubicó en 22,06\% (Instituto Nacional de Estadística - INE, 2016). En los últimos años, el proyecto Global Entrepreneurship Monitor (GEM) ${ }^{4}$ ha encontrado que el porcentaje de emprendedores que crean empresas por la dificultad de acceder al mercado de trabajo, es decir,

4 El Proyecto GEM (Global Entrepreneurship Monitor) fue creado en 1997 con el propósito fundamental de estudiar la relación entre la creación de empresas, o lo que en terminología anglosajona se conoce como entrepreneurship, y el crecimiento económico. Es considerado en la actualidad como el mayor proyecto de investigación existente en el tema del espíritu empresarial, tanto por su magnitud mundial como por sus resultados (Reynolds et al., 2002). En números GEM es: 17 años de datos, más de 200.000 entrevistas por año, más de 100 países, más de 500 especialistas en investigación sobre emprendimiento, más de 300 instituciones académicas y de investigación involucradas, más de 200 instituciones de financiación (Global Entrepreneurship Monitor - GEM Consortium, 2016). 
vinculadas a la necesidad, ha ido en aumento, y en algunos casos estas empresas, no son muy competitivas, pues con frecuencia sus propietarios carecen de habilidades que les permitan expandir sus negocios (Global Entrepreneurship Monitor - GEM Consortium, 2016a).

Extremadura no es una región ajena a la situación de desempleo española, de hecho, en el 2015 su tasa de paro se situó en 29,09\% (INE, 2016), siendo de las más altas entre todas las comunidades autónomas (CCAA). A este hecho, se le asocian otros problemas, como ser una región fundamentalmente rural y considerada como la menos desarrollada en España, así como por tener una baja densidad de población (26 hab/ $\mathrm{km}^{2}$ ). Según datos del Instituto de Estadística de Extremadura (IEEX) en el 2015 y por cuarto año consecutivo la región pierde población, alcanzando una cifra de seis mil extremeños menos, comparado con el 2014 (IEEX, 2016).

En términos económicos, para el 2011, la tasa de crecimiento del producto interno bruto (PIB) de Extremadura en términos reales fue la peor del país, con un $0,9 \%$ de decrecimiento. Adicionalmente, el PIB per cápita es el más bajo de España y a nivel de la Unión Europea (UE) se ubica por debajo del 75\% de la media. Este panorama refleja que el desempleo es en parte el responsable de la situación problemática de la CA, y, por tanto, es necesario incentivar el desarrollo del territorio a través del emprendimiento (Hernández et. al., 2013), pues superar estos problemas supone un interés vigente para mejorar la calidad de vida de la CA, y pueden contribuir a contener la degradación del territorio, afectado de forma importante por el éxodo rural y la recesión.

El emprendimiento es importante para el desarrollo de los territorios, ya que la prosperidad económica y social depende en parte de la dinámica emprendedora (Amorós y Poblete, 2013). Si bien es cierto que no todos los emprendimientos llegan a ser exitosos, sus actividades pueden tener un efecto beneficioso en las economías, pues el ingreso de nuevos competidores, productos y servicios puede ejercer presión sobre empresas establecidas para mejorar su desempeño (Amorós y Poblete, 2013).

El surgimiento de iniciativas emprendedoras puede relacionarse con los ecosistemas de emprendimiento, pues estos ofrecen condiciones que permiten o inhiben la creación de nuevas empresas (Banco Interamericano de Desarrollo - BID, 2007). Según el WEF (2009), es importante conocer el ecosistema de emprendimiento, sus componentes, cómo se comportan y cómo interactúan, pues este entendimiento permite sugerir prácticas para mejorar el proceso, planificar alternativas y ofrecer información de importancia a los líderes del gobierno para estimularlo (Hernández y González, 2015). Igualmente, cada vez más investigadores, tratan de averiguar en qué medida son determinantes los factores del entorno y cómo deben moldearse para favorecer un entorno más propenso a la creación empresarial y la generación de empleo (Hernández et al., 2013). 
Parece ser que la existencia de un ecosistema de emprendimiento propicio para el surgimiento de iniciativas empresariales puede ayudar a solucionar los diversos problemas que afectan a los territorios; en el caso de Extremadura, conocer el ecosistema de emprendimiento rural cobra especial interés, puesto que un buen ecosistema incidiría positivamente en mejorar las condiciones de empleabilidad de la región y el PIB per cápita, elementos que ayudarían a frenar problemas como la despoblación rural o la masculinización y de este modo, mejorar la calidad de vida de los habitantes y dinamizar los espacios rurales de la CA.

Ante este panorama, el objetivo de este trabajo fue identificar y analizar cuáles fueron los factores del entorno que impulsaron o dificultaron la actividad emprendedora rural en Extremadura durante el período 2003 - 2012, mediante los datos recolectados por el proyecto GEM Extremadura.

La aportación fundamental de la investigación ha consistido en desvelar cuáles han sido, en los diez años analizados, las condiciones del entorno emprendedor que más han impulsado y obstaculizado la actividad emprendedora. A partir de aquí, los decisores públicos tienen elementos objetivos de juicio para tomar decisiones que puedan incidir en mejorar las condiciones del entorno e impulsar la actividad emprendedora en Extremadura en los próximos años.

\section{Metodología}

El enfoque de la investigación fue de carácter descriptivo, puesto que se recopilaron datos para describir una situación a partir de diferentes cuestiones, y de las cuales se obtuvo información para realizar la investigación (Cubo, 2011). A partir de estos datos, se hizo un análisis de las condiciones del entorno que incidieron en el ecosistema emprendedor rural de Extremadura y se buscó aportar información sobre las condiciones que favorecieron o limitaron el emprendimiento en el período 2003 - 2012.

Para realizar este trabajo se emplearon fuentes secundarias de información, provenientes principalmente de los datos del proyecto GEM Extremadura, así como de la consulta de libros, artículos científicos, informes, entre otros. Se eligió el proyecto GEM, puesto que ofrece datos del ecosistema emprendedor rural de Extremadura, y, es el proyecto más grande que estudia el espíritu empresarial y el entorno del fenómeno emprendedor (Díaz, 2003; Reynolds, Hay y Camp, 2002); y, también, porque su modelo teórico, parte del supuesto de que la creación de empresas incide en el crecimiento económico (Urbano, Rojas y Díaz, 2010), afirmación importante para Extremadura, donde se necesita impulsar el crecimiento económico, la empleabilidad y la generación de empresas sobre todo en las áreas rurales. El período de tiempo estudiado se estableció en razón a que los datos de entorno rural necesarios para realizar el trabajo solo estaban disponibles hasta el año 2012. 
La información utilizada en este trabajo provino de la encuesta a los expertos en el tema para emprender (National Experts Survey - NES) durante el período 2003 a 2012 en el entorno rural y urbano de Extremadura. El GEM realiza esta encuesta a expertos que habitan o trabajan en la región que se va a estudiar, $\mathrm{y}$, usan variables secundarias procedentes de diversas fuentes, como el Fondo Monetario Internacional (FMI), la Organización para la Cooperación y el Desarrollo Económico (OCDE), la Organización de las Naciones Unidas (ONU), la Unión Europea (UE), entre otros (Fernández, Peña, Guerrero y González, 2014), con el fin de valorar su influencia en el proceso emprendedor y condicionar el ecosistema de emprendimiento.

La encuesta NES se aplica a 36 expertos (4 por cada condición del entorno, a través de un enfoque de muestra de conveniencia) en nueve temas (apoyo financiero, políticas gubernamentales, programas gubernamentales, educación y formación emprendedora, transferencia de I+D, acceso a infraestructura comercial y profesional, apertura del mercado interior, acceso a infraestructura física y de servicios, y normas sociales y culturales) (GEM Consortium, 2016); sin embargo, el GEM puede obtener valoraciones de condiciones (EFCS) adicionales. En este trabajo, se incluyeron siete EFCS adicionales que fueron percepción de oportunidades, habilidades para emprender, motivación para emprender, legislación sobre propiedad intelectual, apoyo a la mujer emprendedora, apoyo al crecimiento e innovación, puesto que podían ofrecer información relevante sobre otros factores que pueden influir en el ecosistema emprendedor rural de Extremadura.

La información de la encuesta NES facilitada por el proyecto GEM Extremadura, fue organizada en Microsoft Excel ${ }^{\circledR}$; posteriormente, se elaboraron gráficos que permiten mostrar series de datos numéricos y facilitan su comprensión, y de este modo, permitieron comparar y analizar la información del entorno rural con el entorno urbano de Extremadura. Se hizo un gráfico de barras para mostrar las valoraciones realizadas por los expertos sobre las condiciones que influyeron en el ecosistema emprendedor rural de la CA. Para comparar, analizar y concluir cuáles condiciones del entorno facilitaron y obstaculizaron el emprendimiento, se diseñó un gráfico de líneas.

\section{Resultados}

Valoración general de las condiciones del ecosistema emprendedor rural en Extremadura durante el periodo 2003 - 2012

La figura 1 muestra la valoración realizada por los expertos rurales sobre las condiciones específicas del entorno emprendedor en Extremadura, estos valores brindan una visión general del comportamiento del ecosistema emprendedor rural de la CA y de aquellos factores que pudieron incidir en la actividad emprendedora de la región. 
Figura 1. Valoración media de las condiciones específicas del entorno rural para emprender en Extremadura durante el período 2003 - 2012

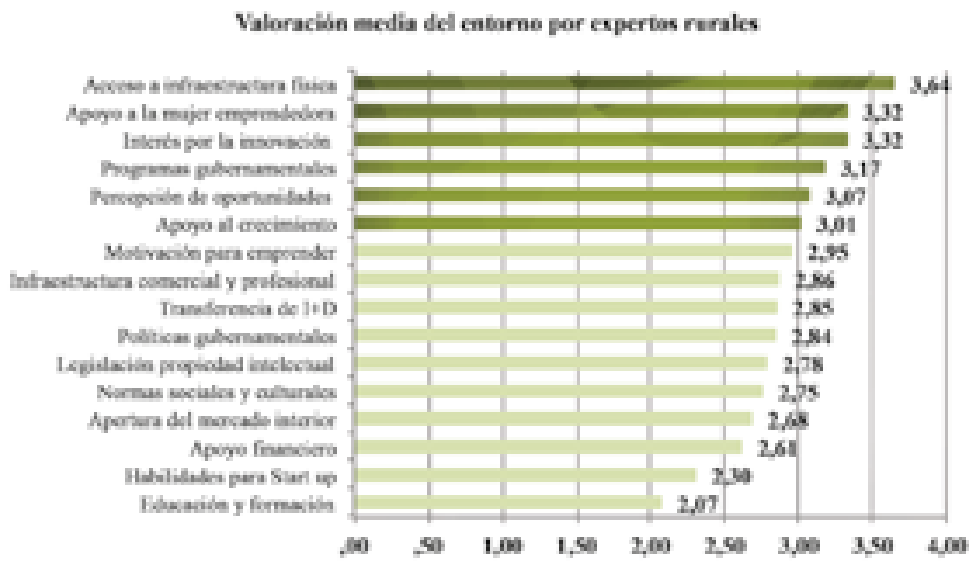

Fuente. Elaboración propia a partir de los datos del Proyecto GEM Extremadura.

La figura 1 muestra que el acceso a la infraestructura física, el apoyo a la mujer emprendedora, el interés por la innovación, los programas gubernamentales, la percepción de oportunidades y el apoyo al crecimiento fueron las únicas condiciones que al parecer favorecieron las tasas de actividad emprendedora en el entorno rural de la CA. En contraparte, los expertos señalaron que la educación y la formación, las habilidades para emprender y el apoyo financiero fueron las condiciones más limitantes para fomentar el emprendimiento en el entorno rural.

De manera general, estos resultados (figura 1) dejan entrever que las condiciones del entorno no fueron las más propicias para emprender en el ámbito rural durante el período estudiado, puesto que más de la mitad de las condiciones valoradas no superan el valor medio (3.00). De alguna forma, los resultados no sorprenden y se pueden vincular con diferentes sucesos, como la reciente crisis financiera que sufrió Europa, que inició en el 2008 y que comenzó a superarse hacía el 2012, la cual pudo agudizar problemas como el acceso a la financiación; en palabras de la UE:

En general, los emprendedores potenciales se encuentran en Europa en un entorno difícil, la educación no ofrece una base sólida para iniciar la carrera empresarial, hay dificultad para acceder al crédito y a los mercados, problemas para traspasar negocios, miedo a sanciones en caso de fracaso y onerosos procedimientos administrativos (Comisión Europea, 2012, p. 4).

Los resultados relacionados con la educación para el emprendimiento dejan ver que es un aspecto de especial interés por mejorar; las políticas y lineamentos establecidos desde la UE señalan que se ha venido trabajando al respecto, por ejemplo, como lo evidencia lo establecido en la Carta Europea de la Pequeña Empresa, y continuará tra- 
bajándose por mejorarlo como lo indica la Estrategia Europa 2020 (Comisión Europea, 2010). Desde el año 2000, mediante la Carta Europea de la Pequeña Empresa, Europa se propuso educar en el espíritu empresarial y las nuevas habilidades desde una edad temprana, es decir, enfatizó que en todos los niveles escolares deben transmitirse conocimientos sobre la actividad y el espíritu empresarial; asimismo, motivó la creación de módulos sobre temas empresariales, que constituyan un elemento fundamental de los programas educativos de la enseñanza secundaria y superior (Comisión Europea, 2000). En España, con el fin de asumir los lineamientos del Consejo Europeo de Lisboa para promover el espíritu emprendedor en el sistema educativo, se introdujo la Ley 10/2002 o Ley Orgánica de Calidad de la Educación (LOCE), la cual establece en uno de sus principios de calidad "la capacidad de los alumnos para confiar en sus propias aptitudes y conocimientos, desarrollando los valores y principios básicos de creatividad, iniciativa personal y espíritu emprendedor" (Boletín Oficial del Estado - BOE, 2002).

De igual forma, en el Plan de Acción sobre Emprendimiento 2020, se establece un programa para facilitar la creación de empresas y crear un entorno más favorable, en el cual los emprendedores puedan prosperar; este plan fomenta acciones para liberar el potencial del emprendimiento, eliminar los obstáculos existentes y revolucionar la cultura del emprendimiento en Europa (Comisión Europea, 2012) y propone tres áreas de intervención, que son:

1. Educar y formar en materia de emprendimiento para promover el crecimiento y la creación de empresas; 2 . Reforzar las condiciones marco para los emprendedores, eliminando barreras estructurales y prestándoles apoyo en las fases cruciales del ciclo vital de la empresa; y, 3. Dinamizar la cultura del emprendimiento en Europa: crear una nueva generación de emprendedores (Comisión Europea, 2012, p. 6).

En cuanto a las condiciones mejor valoradas por los expertos, puede inferirse que se corresponden con el nivel de desarrollo alcanzado por los países europeos. Podría decirse que el acceso a la infraestructura física se encuentra en un punto óptimo de desarrollo, que permite que los emprendimientos generados tengan un buen acceso a infraestructuras de comunicación y de servicios básicos, que les facilita acercarse a los mercados y mantener una producción adecuada a sus necesidades. El apoyo a la mujer emprendedora y el interés por la innovación, han sido aspectos favorecidos por programas estratégicos que buscan incentivar su crecimiento. El caso de la mujer adquiere relevancia, puesto que representa el 52\% de la población europea total, por tanto, la Comunidad Europea (CE) quiere apoyar al máximo el espíritu empresarial femenino e impulsar líneas de tutoría, asesoramiento, educación y plataformas de redes de negocios para mujeres empresarias (Comisión Europea, 2013).

Del mismo modo, los resultados obtenidos son consistentes con los del proyecto GEM España y GEM Extremadura en el año 2014, en los cuales también se deduce que el entorno continúa siendo poco favorable para iniciar negocios, y se evidencia que entre las condiciones mejor valoradas están el acceso a la infraestructura física, la existencia de infraestructura comercial y profesional y los programas guber- 
namentales. En contrapartida, en dicho año, las condiciones peor valoradas siguen siendo la educación y formación emprendedora y el apoyo financiero (Fernández et al., 2014; Hernández et. al., 2014). Por tanto, las valoraciones obtenidas en el entorno rural siguen siendo consistentes con el panorama más actual de la CA y del país, y es necesario continuar trabajando en mejorar el ecosistema emprendedor, puesto que el emprendimiento, como ya se mencionó, favorece el crecimiento económico de las regiones, ayuda a disminuir las tasas de desempleo y puede ser una alternativa para frenar la despoblación de las áreas rurales de la CA.

\section{Comparación de las condiciones del entorno que influyeron en el} ecosistema emprendedor rural versus el ecosistema emprendedor urbano en Extremadura durante el periodo 2003 - 2012

Para observar el comportamiento de las condiciones del entorno a nivel rural y urbano, se hizo una comparación de las valoraciones realizadas por los expertos. La comparación muestra que, tanto en el entorno rural como en el urbano, seis condiciones favorecieron el ecosistema de emprendimiento para iniciar nuevos negocios (figura 2). Cinco de las condiciones mejor valoradas (percepción de oportunidades, programas gubernamentales, interés por la innovación, apoyo a la mujer emprendedora y acceso a infraestructura física) fueron comunes tanto en el entorno rural como en el urbano. Sin embargo, la infraestructura comercial y profesional fue mejor valorada en el entorno urbano, quizás porque en estos espacios hay mayor presencia de universidades y centros educativos, $\mathrm{y}$, por ende, se encuentran más profesionales (públicos o privados) para dar soporte a las necesidades de los emprendedores, por ejemplo, de índole legal, contable, financiera, etc., en cualquier etapa de la actividad empresarial.

Figura 2. Comparativo de la valoración media de los expertos sobre las condiciones del entorno rural versus el urbano para el emprendimiento

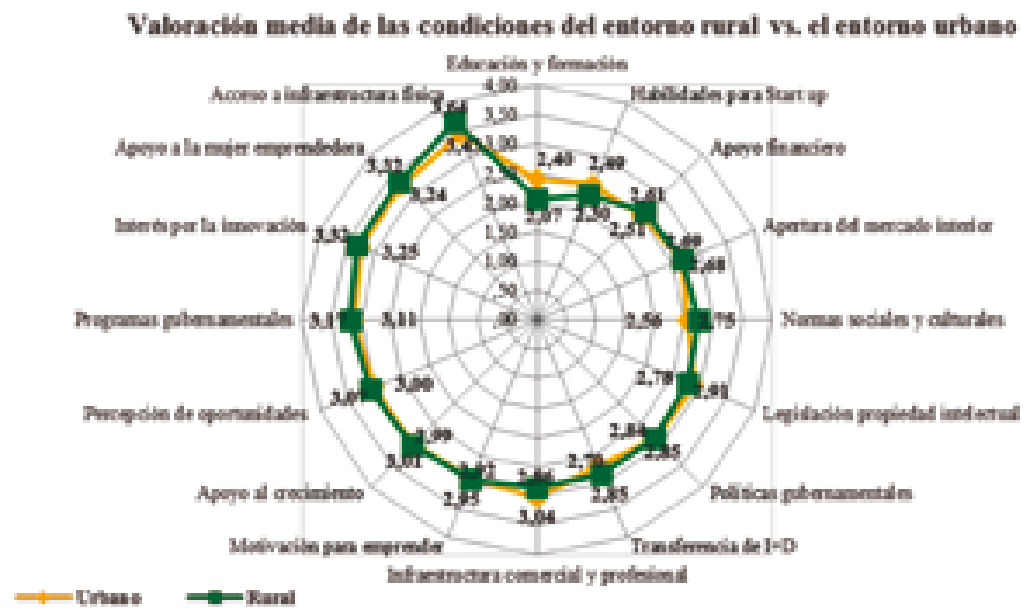

Fuente. Elaboración propia a partir de los datos del Proyecto GEM Extremadura. 
A nivel urbano y rural, las condiciones del entorno peor valoradas fueron la educación y formación, las habilidades para start up (emprender) y el apoyo financiero; no obstante, la educación y formación fue peor valorada en el entorno rural y se ubicó como la condición peor valorada del período. Estos resultados confirman que la educación y el apoyo financiero, requieren atención especial, pues al parecer condicionaron el emprendimiento en la CA.

En cuanto a las condiciones del entorno peor valoradas, la condición educación y formación, hace referencia a la medida en la que los sistemas educativos y de formación incorporan herramientas para formar en creación, gestión y dirección de nuevas empresas.

La baja valoración de esta condición puede explicarse por varias circunstancias; por una parte, Extremadura cuenta con bajos niveles de población, y esta situación se agudiza principalmente en las áreas rurales, razón que ha generado una menor oferta de servicios educativos en los municipios, por lo cual, los niños y jóvenes deben desplazarse a lugares cercanos a su residencia para recibir formación primaria, secundaria y posecundaria, lo cual puede haber incidido en abandono escolar. Adicionalmente, suele ser común que los habitantes de las áreas rurales tengan menores niveles educativos que los habitantes del entorno urbano, probablemente relacionado con la menor oferta educativa.

Sumado a esto, en Extremadura solo existe una universidad de carácter público, que cuenta con cuatro espacios de formación ubicados en Badajoz, Cáceres, Mérida y Plasencia, donde se ofrecen programas de grado, posgrado, doctorado, títulos propios y formación continua. A la fecha estudian más de 24000 alumnos, teniendo en cuenta que la CA tiene alrededor de 1100000 habitantes, podría indicarse, que un bajo porcentaje de la población accede a estudios de postsecundaria, lo cual puede repercutir en la escasa formación para el emprendimiento en la CA (Universidad de Extremadura - UEX, 2016).

Por otra parte, desde hace algunos años a nivel europeo se ha generado normatividad que regula la incorporación de estrategias y herramientas en el sistema educativo en todos los niveles (primaria, secundaria y postsecundaria) para formar en creación, gestión y dirección de nuevas empresas, aumentar el espíritu emprendedor y fomentar la cultura emprendedora; dentro de las directrices existentes se pueden resaltar las siguientes: el Consejo Europeo de Lisboa en el año 2000; el Informe Educación y Formación en el Espíritu Empresarial en el año 2002; el Libro Verde: el Espíritu Empresarial en Europa en el año 2003; la recomendación del Parlamento Europeo y el Consejo en el año 2006, en su página L394/13; la Small Business Act de la Comisión de las Comunidades Europeas en el año 2008; y, para terminar, en el año 2013, el Plan de Acción sobre Emprendimiento 2020 de la CE. A nivel de Extremadura. También existe un marco normativo para fomentar el espíritu emprendedor en la educación; este marco está compuesto por la Ley 4/2011 o Ley de Educación, que en su artículo 81 establece a la capacidad emprendedora como aspecto prioritario 
del currículo (BOE, 2011); la orden de 24 de mayo que establece la obligatoriedad de ofertar una materia relacionada con la iniciativa emprendedora en la educación secundaria (DOE, 2007); la orden de 31 de mayo donde se creó la Red Extremeña de Escuelas Emprendedoras (DOE, 2011); y en el 2012 los decretos 108/2012 (DOE, 2012) y 109/2012 (DOE, 2012a) (Junta de Extremadura, 2016), en los cuales se establece el emprendimiento como parte del currículo en la educación primaria y en la educación secundaria obligatoria.

Si bien, esto refleja la existencia de un marco normativo, los resultados señalan la necesidad de evaluar la efectividad de este. De igual forma, se sugiere generar programas específicos de formación para el emprendimiento que contribuyan a mejorar las tasas de actividad emprendedora en la CA; los avances a nivel de telecomunicaciones pueden potencializar las herramientas encaminadas a favorecer el emprendimiento y que estas lleguen de una manera más amplia y eficaz a la población sobre todo a la ubicada en áreas rurales.

Las habilidades para emprender fueron la condición con la segunda peor valoración realizada por los expertos, principalmente en el entorno rural. Esta condición hace referencia a la capacidad que posee la población para crear y desarrollar nuevas empresas (Hernández et al., 2013). El resultado obtenido revela la necesidad de generar estrategias para que la población adquiera o mejore las habilidades para iniciar nuevos negocios; de igual forma, se puede inferir que, a nivel rural, la población cree tener menos habilidades para iniciar un negocio que la población urbana, lo cual podría relacionarse con la menor oferta educativa que hay en estas áreas, la menor disponibilidad de experiencia en diferentes sectores productivos, $\mathrm{y}$, de relacionamiento con emprendedores.

Este panorama enuncia que el fomento de políticas de espíritu empresarial relacionadas, por ejemplo, con la educación en emprendimiento es un buen camino por seguir. En palabras de Stevenson (2002) existen tres tipos de destrezas o de habilidades que ayudan a fomentar el emprendimiento, que son las destrezas administrativas, técnicas y empresariales, por lo cual las políticas de espíritu empresarial deben estar basadas en la destreza, y para mejorar la base de estas destrezas y que la gente se convierta en empresaria, es necesario impulsarlas mediante la educación. Vale la pena resaltar que en España la Ley 14/2013, de apoyo a los emprendedores y su internacionalización, incorpora medidas al sistema educativo para que los jóvenes adquieran competencias y habilidades para emprender (BOE, 2013).

En síntesis, no solo las condiciones económicas son necesarias para que el ecosistema de emprendimiento sea favorable e influya positivamente en el aumento de las tasas de actividad emprendedora; también es necesario que exista población que confíe en sus capacidades y se incline a iniciar nuevos negocios, que generen empleo y desarrollo en los lugares donde se ubicarán, pues es con la combinación de ideas de negocio, habilidades, recursos financieros y no financieros, como se pueden generar empresas dinámicas (Auletta y Rivera, 2011). De igual forma, se deben incentivar 
los emprendimientos por oportunidad a nivel rural y urbano, pues en estos, el emprendedor tiene una visión clara del negocio y de las habilidades para llevarlo a cabo (Amorós y Bosma, 2014), a diferencia de los emprendimientos por necesidad, donde no suele haber una visión, ni hay confianza en las habilidades para alcanzarla (Díaz y Cancino, 2014).

El tercer obstáculo para emprender a nivel rural y urbano fue el apoyo financiero; este, se refiere a la disponibilidad de recursos financieros, capital y deuda, para las empresas nuevas y en crecimiento (Pyme), incluyendo subvenciones y subsidios (GEM Consortium, 2016; Hernández y Díaz, 2005).

Esta baja valoración puede relacionarse con la falta de capital propio, acceso a financiación de entidades financieras, de inversores privados o de capital riesgo para impulsar nuevas iniciativas empresariales y la baja salida a la bolsa. De igual forma, puede asociarse a la crisis económica que afectó a Europa, pues esta pudo condicionar la aparición de nuevas empresas y actuar como variable de freno para mejorar las tasas de actividad emprendedora en la CA a nivel rural y urbano. Asimismo, las valoraciones obtenidas coinciden con la información que presenta el Libro verde. El espíritu empresarial en Europa, donde se afirma que el acceso a la financiación es un obstáculo para los nuevos empresarios, pues no tienen facilidad para conseguir préstamos bancarios y capital riesgo (Comisión Europea, 2003). Por el contrario, las subvenciones públicas fueron la única fuente de recursos que al parecer incentivó el emprendimiento en la CA.

En contraparte, las condiciones que favorecieron el entorno emprendedor en la CA fueron, el acceso a la infraestructura, el apoyo a la mujer emprendedora y el interés por la innovación.

El acceso a la infraestructura física fue la condición mejor valorada a nivel rural y urbano. La valoración de los expertos permite inferir que, durante el período estudiado, el nivel de desarrollo en infraestructura física era adecuado y los emprendedores contaban con acceso a infraestructuras físicas (carreteras, telecomunicaciones, etc.), servicios básicos (gas, agua, electricidad, etc.) y sistemas de comunicación (teléfono, internet, etc.), pues podían acceder a estos en poco tiempo y afrontar los costos que suponían. Si bien se suele suponer que emprender en el entorno rural supone mayores dificultades por causa del aislamiento o por tener menor infraestructura que el entorno urbano, al parecer durante el período estudiado en la CA, las áreas rurales poseían una infraestructura física que no frenaba el emprendimiento.

De igual manera, estos resultados señalan que las decisiones político-administrativas, son consecuentes con las necesidades de los emprendedores y que orientan esfuerzos para moldear un ecosistema de emprendimiento más propicio para el desarrollo de iniciativas emprendedoras; por ejemplo, algunas políticas o lineamentos de la UE muestran que esta condición ha dejado de ser un tema prioritario, puesto que el nivel de desarrollo de infraestructura física es óptimo y por tanto no 
representa obstáculos para emprender, y hay temas con mayor relevancia actual para mejorar las tasas de actividad emprendedora, a los cuales hay que dedicar mayor atención, como es el caso de la educación y la formación, la innovación, el acceso a la financiación, entre otros, como se puede observar en el documento Plan de Acción sobre Emprendimiento 2020. Relanzar el espíritu emprendedor en Europa (Comisión Europea, 2012).

No obstante, es necesario mantener y mejorar la infraestructura física en aquellas zonas de la CA donde el acceso a algunos servicios aún puede ser limitado, puesto que el emprendimiento en estas áreas ayudaría a mitigar la despoblación rural, atraer nueva población y de este modo dinamizarlas. Ante esto, es interesante resaltar que dentro del programa de desarrollo rural (PDR) de Extremadura 2014 - 2020 se incluyen medidas que buscan modernizar y desarrollar tecnológicamente las explotaciones, mejorar la red de caminos y la red viaria; generar empleo; mantener y mejorar el tejido empresarial; crear actividades generadoras de riqueza en el medio rural, como actividades turísticas y medioambientales; en resumen, buscan mejorar y desarrollar las infraestructuras, diversificar y dinamizar la economía, y, fomentar el cooperativismo rural (Junta de Extremadura, 2015).

La segunda condición mejor valorada fue el apoyo a la mujer emprendedora. Esta condición se refiere a la percepción que existe en cuanto a las habilidades y capacidades que posee la mujer para emprender y a las medidas de apoyo para su fomento (políticas, servicios sociales, normas sociales, etc.) (Hernández y Díaz, 2005). Las valoraciones obtenidas enuncian que existió una situación favorable para que la mujer emprendiera o se autoempleara en la CA; de igual forma, señalan que las mujeres tenían (en teoría) habilidades y capacidades para crear nuevas empresas, y, se habían impulsado diversos programas y medidas de apoyo en la CA para soportar tal fin.

En contraparte, al parecer, el único aspecto que no favoreció la condición a nivel rural y urbano fue la baja disponibilidad de servicios sociales para que las mujeres continuaran trabajando después de haber formado una familia. Esta situación coincide con la información presentada en el Plan de Acción sobre Emprendimiento 2020 - Relanzar el espíritu emprendedor en Europa, el cual menciona que una de las principales dificultades que encuentra la mujer para emprender, es la conciliación de la actividad empresarial con la familiar; por tanto, es prioritario promover más servicios sociales, que les permitan conciliar estas dos actividades (Comisión Europea, 2012).

Dentro de los programas que se desarrollaron en el período estudiado en la CA y que pueden estar relacionados con la buena valoración, se encuentran los siguientes: el Plan de Fomento y Calidad del Empleo de Extremadura, que incluía medidas para favorecer las iniciativas emprendedoras de las mujeres, contemplaba acciones para conciliar la vida profesional y privada y mejorar la oferta formativa (Junta de Extremadura, 2008). Otro programa impulsado en la CA fue "Es tu Oportunidad Mujer Emprendedora”, promovido por el Ministerio de Sanidad, Política Social e Igualdad, 
dentro del Programa Especial para Extremadura y la Consejería de Igualdad y Empleo de la Junta de Extremadura, a través del Servicio Extremeño Público de Empleo (SEXPE) y Fomento de Emprendedores en el año 2010, cuyo propósito fue disminuir el desempleo femenino, fomentar el espíritu empresarial de mujeres interesadas en iniciar su propia empresa (Junta de Extremadura, 2010a).

En el 2011 la Junta de Extremadura creó el Consejo Extremeño de Participación de las Mujeres, el cual es un órgano consultivo y de participación de las asociaciones y organizaciones de mujeres y los agentes sociales y económicos en las políticas de igualdad de género (Junta de Extremadura, 2011).

En años posteriores al período estudiado, dentro de la CA se han impulsado nuevas medidas para favorecer el emprendimiento femenino, como es el caso de la estrategia "Ser Empresaria", impulsada en el 2013, que busca visibilizar a las empresarias, aumentar el emprendimiento femenino y ayudar a sensibilizar el mundo empresarial en igualdad de género. Este programa contempla medidas de apoyo económico y asesoramiento dirigidas a conciliar la vida laboral, personal y familiar, formación en liderazgo, creación de empresas, gestión empresarial; además, fomenta la cultura emprendedora desde edades tempranas y desarrolla programas para el uso de las tecnologías de la información y la comunicación (TIC) (Junta de Extremadura, 2013).

En conclusión, es importante mantener e impulsar programas, medidas y servicios para que las mujeres continúen emprendiendo, puesto que constituyen la mayor parte de la población europea y, por tanto, para mejorar el crecimiento económico, incidir positivamente en la generación de empleo y riqueza, sobre todo en aquellas áreas más desfavorecidas de la CA es prioritario vincular a la mujer (Comisión Europea, 2012).

A nivel urbano y rural, la tercera condición del entorno mejor valorada fue el interés por la innovación; condición que analiza el interés desde el punto de vista de las nuevas empresas y de los consumidores.

La buena valoración de la innovación señala que las nuevas empresas estaban abiertas a introducir procesos innovadores y nuevas tecnologías en los emprendimientos. Cabe señalar que incluir la innovación en los emprendimientos es tendencia a nivel mundial, sobre todo en países con altos niveles de ingresos, como es el caso de España, donde se sugiere que las iniciativas de emprendimiento deben enfocarse a desarrollar los sectores empresariales con altos niveles de innovación y a apoyar a las nuevas empresas con alto valor añadido que tienen el potencial para crecer y desarrollarse internacionalmente (Hernández y Díaz, 2005).

De igual forma, los resultados señalan que los consumidores valoraban de manera positiva la innovación, es decir, que estaban interesados y dispuestos a comprar productos y servicios innovadores, quizás porque estos ayudan a satisfacer las necesidades que los nuevos modos de vida y de relacionamiento social van imponiendo. De manera general, estos resultados son interesantes en la medida en que los em- 
prendedores consideran la innovación como una herramienta útil y necesaria para introducir en sus iniciativas; $y$, a su vez, porque existe un mercado propicio para introducir nuevos productos y servicios, pues los consumidores esperan su oferta. Estos resultados coinciden con los obtenidos en España, donde se considera a la innovación como uno de los principales facilitadores de la actividad empresarial (GEM Consortium, 2016a).

A pesar de que durante el período estudiado se obtuvo un resultado favorable en la CA sobre esta condición, para seguir fortaleciendo el entorno en materia de innovación, en el 2013 se creó el Centro Europeo de Empresas e Innovación (CEEI) Extremadura, organización que promueve, estimula y desarrolla la innovación en pequeñas y medianas empresas (Pymes) en todas las etapas de su ciclo de vida. El CEEI busca potenciar la creación de empresas de innovación y desarrollarla en las existentes, para contribuir al desarrollo económico, la competitividad y el crecimiento de la región. Este programa es especial, pues busca dar atención prioritaria a las áreas rurales y llevar sus servicios a toda la población para promover el desarrollo integral del territorio.

El CEEI Extremadura presta servicios relacionados con motivación a emprendedores, identificación de oportunidades de negocio, asesoramiento personalizado, formación, financiación, entre otros, que buscan que las empresas extremeñas constituyan un tejido productivo diversificado, especializado y con capacidad de competir en mercados globales (Centro Europeo de Empresas e Innovación - CEEI Extremadura, 2016).

A pesar de este panorama, es necesario mantener y generar más programas de apoyo a emprendedores para que incluyan la innovación en sus nuevos negocios y de esta manera respondan a las necesidades de los consumidores de la CA y del mundo actual.

Los programas gubernamentales fueron otra condición a favor del ecosistema de emprendimiento en la $\mathrm{CA}$, y que al parecer incentivó el inicio de nuevos negocios a nivel urbano y rural, a pesar de la crisis económica. Algunos de los programas que pueden sustentar estos buenos resultados son, por ejemplo: Extremadura Avante, S.L.U. (Junta de Extremadura, 2010), el programa para el emprendimiento y la innovación social en Extremadura (Gobierno de Extremadura, 2014), y el Plan de acción integral de empleo, emprendedores y empresa - Plan 3E (Gobierno de Extremadura, 2013a). Si bien existen programas de apoyo a la actividad emprendedora en la región, es necesario mejorar la efectividad de los mismos, pues estos fortalecen a la empresa como institución generadora de riqueza y desarrollo, están comprometidos con el cambio de modelo productivo que necesita la CA para su avance, permitirían disminuir las tasas de desempleo actuales y así frenar los problemas de despoblación que aquejan sobre todo las áreas rurales de la CA.

La percepción de oportunidades también favoreció el entorno emprendedor, sin embargo, al parecer la crisis económica perjudicó la apreciación que tenían los em- 
prendedores en el entorno urbano sobre las oportunidades existentes en el mercado para iniciar nuevos negocios; situación probablemente asociada con el estancamiento de las condiciones económicas de la región. Por otra parte, podría inferirse que en el entorno rural la percepción de oportunidades suele estar más ligada al contexto donde se ubicará el negocio y el momento por el que está pasando el mismo, sin tener en cuenta, en gran medida, las condiciones macroeconómicas que puedan afectarle, como sí puede suceder en el entorno urbano. Debe tenerse en cuenta que la percepción de oportunidades suele relacionarse con el entorno, es decir, aunque existan personas motivadas y quieran convertirse en empresarios, si la estructura de la sociedad y la del Gobierno no es adecuada, así como las condiciones económicas no son favorables, es difícil lograrlo (Stevenson, 2002).

\section{Conclusiones y discusión}

El término ecosistema emprendedor se utiliza para ofrecer un panorama de las condiciones del entorno que favorecen y obstaculizan el proceso emprendedor en un país o región a nivel rural o urbano (Fernández et al., 2014). El estudio realizado permite señalar que las condiciones que favorecieron y limitaron el emprendimiento a nivel rural fueron coincidentes a nivel urbano, por lo cual, parece ser que en el período estudiado los emprendedores de la CA se encontraban con facilidades y obstáculos semejantes para poner en marcha sus emprendimientos.

Los resultados señalan que no solo los aspectos institucionales influyeron en el entorno emprendedor rural de la CA, pues, la baja valoración de las habilidades para emprender evidencia que los factores socioculturales pudieron incidir y obstaculizar la creación de empresas; esto, sugiere la necesidad de impulsar medidas para incrementar la motivación y las habilidades que requieren las personas para emprender en las áreas rurales, así como incentivar políticas de espíritu empresarial que incluyan el área de motivación, oportunidad y destrezas para aumentar el número de nuevos empresarios y nuevas compañías (Stevenson, 2002) y de este modo incrementar las tasas de actividad emprendedora en la CA.

Como lo afirma Stevenson (2002), si es difícil empezar un negocio, conseguir asesoría, obtener información, si hay barreras, altos costos, burocracia, poca financiación y además toma mucho tiempo, entonces va a ser muy difícil crear empresas. Por lo cual, se deben implementar estrategias a nivel rural y urbano que ayuden a disminuir los obstáculos para emprender, así, la inversión y la productividad serán mecanismos que estimulen la creación de riqueza, la diversificación de la actividad económica, y la solución de problemas como el desempleo que afectan principalmente las áreas rurales de la CA (Díaz, 2003). 
Desde hace décadas la UE prioriza la necesidad de crear un mejor entorno para las pequeñas empresas y para mejorar el espíritu emprendedor (Comisión Europea, 2000), sin embargo, es necesario continuar trabajando al respecto y evaluar lo implementado para verificar la efectividad de las acciones. Si bien, en la CA se han implementado estrategias y programas para mejorar el entorno emprendedor, es vital que estos atiendan las necesidades reales de los emprendedores y que su efectividad sea más controlada, con el fin de que estos esfuerzos se traduzcan en más emprendimientos para mejorar las condiciones económicas de la CA.

Las políticas o lineamientos gubernamentales que se generen para favorecer el emprendimiento y aumentar la creación de empresas en el entorno rural, deben actuar eficazmente sobre la educación y formación emprendedora en todos los niveles, y propender porque sea accesible en toda la $\mathrm{CA}$, primando de algún modo el uso de las TIC para alcanzar este fin. De igual forma, es clara la necesidad de mejorar el apoyo financiero para la creación de nuevas empresas o la consolidación de estas. A su vez, se debe fomentar la cultura empresarial, es decir, impulsar medidas para que la población visualice el emprendimiento como una alternativa para mejorar su calidad de vida y la de la población que lo rodea, e incidir positivamente en la disminución de los actuales problemas que aquejan a la $\mathrm{CA}$, como la despoblación, la falta de empleo, la escasa diversificación económica, entre otros.

Todo esto es necesario, puesto que algunas de las prioridades de las áreas rurales de la CA como se menciona en el PDR 2014-2020, hacen énfasis en fortalecer el tejido socioeconómico, mejorar el emprendimiento rural, crear más y mejores empleos, mejorar la imagen social del empresario rural, de manera general, impulsar que la generación de empresas e ideas de negocio en dichas áreas se haga más atractiva para enfrentar de una forma más eficaz los problemas que aquejan a estas áreas (Junta de Extremadura, 2015).

De igual forma, los resultados obtenidos se relacionan con las sugerencias de Stevenson (2002) para la creación de políticas que favorezcan la actitud hacia el espíritu empresarial y el incremento de la actividad emprendedora, pues señala que deben incluir medidas de apoyo no financiero básico, como incubadoras, iniciativas de apoyo; acceso a recursos o financiación; educación en todos los niveles sobre espíritu empresarial; mantener las iniciativas de políticas orientadas a diferentes grupos objetivos de la sociedad, por ejemplo, los jóvenes y las mujeres empresarias; y favorecer la cultura del espíritu empresarial, fomentando la empresa, y también tratar de disminuir el estigma al fracaso. Estas medidas, reflejan la solución a los principales factores de freno que señalaron los expertos. 


\section{Referencias}

Acs, Z.J., y Amorós, J.E. (2008). Introduction: the startup process. Estudios de Economía, 35(2), 121-132.

Auletta, N., y Rivera, C. (2011). Un ecosistema para emprender. Debates Iesa, 16, 12-17.

Amorós, J. E., y Poblete, C. (2013). Aspiraciones de los Emprendedores en Chile y el Mundo 2012. Recuperado de http://negocios.udd.cl/gemchile/files/2014/10/GEMChile-2012-Reporte-Aspiracion-Emprendedora.pdf.

Amorós, J. E. (2013). Global Entrepreneurship Monitor: aspiraciones de los emprendedores en Chile y el mundo 2012. Universidad del Desarrollo. Chile.

Amorós, J. E., y Bosma, N. (2014). Global Entrepreneurship Monitor 2013 - Global Report. Recuperado de http://www.gemconsortium.org/report/48772.

Aspen Network of Development Entrepreneurs. (2013). Entrepreneurial Ecosystem Diagnostic Toolkit. Recuperado de http:/www.aspeninstitute.org/sites/default/ files/content/docs/pubs/FINAL\%20Ecosystem\%20Toolkit\%20Draft_print\%20 version.pdf

Banco Interamericano de Desarrollo (BID). (2007). Guía de emprendimientos dinámicos. Recuperado de https://publications.iadb.org/bitstream/ handle/11319/5977/Guia\%20de\%20emprendimiento\%20MIF.pdf?sequence=1.

Boletín Oficial del Estado - BOE. (2002). Ley 10/2002, de 23 de diciembre, de Calidad de la Educación. Recuperado de https://www.boe.es/boe/dias/2002/12/24/pdfs/ A45188-45220.pdf

Boletín Oficial del Estado - BOE. (2011). Ley 4/2011, de 7 de marzo, de Educación de Extremadura. Recuperado de https://www.boe.es/buscar/pdf/2011/BOE-A-20115297-consolidado.pdf

Boletín Oficial del Estado - BOE. (2013). Ley 14/2013, de 27 de septiembre, de apoyo a los emprendedores y su internacionalización. Recuperado de https://www.boe. es/boe/dias/2013/09/28/pdfs/BOE-A-2013-10074.pdf.

Centro Europeo de Empresas e Innovación (CEEI) Extremadura. (2016). CEEI Extremadura. Recuperado de http://www.ceeiextremadura.com/?page_id=2.

Coduras, A., Urbano, D., Rojas, Á., y Martínez, S. (2008). The Relationship Between University Support to Entrepreneurship with Entrepreneurial Activity in Spain: A Gem Data Based Analysis. International Atlantic Economic Society, 14, 395 406. doi: 10.1007/s11294-008-9173-8. 
Comisión Europea. (2000). Carta europea de la pequeña empresa. Recuperado de http://www.ipyme.org/es-ES/UnionEuropea/UnionEuropea/PoliticaEuropea/ Marco/Paginas/Cartaeuropeadelapeque $\% \mathrm{C} 3 \%$ Blaempresa.aspx

Comisión Europea. (2003). Libro verde - El espíritu empresarial en Europa. Recuperado de http://eur-lex.europa.eu/legal-content/ES/TXT/ $\mathrm{PDF} /$ ?uri=CELEX:52003DC0027\&from=ES.

Comisión Europea. (2010). Europa 2020 - Una estrategia para un crecimiento inteligente, sostenible e integrador. Recuperado de https://eur-lex.europa.eu/ legal-content/ES/TXT/PDF/?uri=CELEX:52010DC2020\&from=ES

Comisión Europea. (2012). Plan de acción sobre emprendimiento 2020 - Relanzar el espiritu emprendedor en Europa. Recuperado de http://eur-lex.europa.eu/ LexUriServ/LexUriServ.do?uri=COM:2012:0795:FIN:es:PDF

Comisión Europea. (2012a). Hacia una recuperación generadora de empleo. Recuperado de http://eur-lex.europa.eu/LexUriServ/LexUriServ. do?uri=COM:2012:0173:FIN:ES:PDF

Comisión Europea. (2013, 14 de enero). Europa necesita más emprendedores y la CE ha presentado un plan para apoyarles. Actualidad y Prensa CE. Recuperado de http://ec.europa.eu/spain/actualidad-y-prensa/noticias/empleo-y-politica-social/ plan-emprender_es.htm

Cubo, S. (2011). La investigación experimental. En Cubo, S., Domínguez, E., Luengo, R., Martín, B., y Ramos, J.L. (Eds). Métodos de investigación y análisis de datos en ciencias sociales y de la salud (pp. 235-326). Ediciones Pirámide: Madrid.

Díaz, J.C. (2003). La creación de empresas en Extremadura. Un análisis institucional (tesis doctoral). Universidad de Extremadura, Cáceres, España.

Díaz, D., y Cancino, C. (2014). De emprendimientos por necesidad a emprendimientos por oportunidad: casos rurales exitosos. Multidisciplinary Business Review, 7(1), 48-56.

Díaz, J.C., Hernández, R., Sánchez, M.C., y Postigo, M.V. (2010). Actividad emprendedora y género. Un estudio comparativo. Revista Europea de Dirección y Economía de la Empresa, 19(2), 83-98.

Díaz, J.C., Pulido, U., y Hernández, R. (2005). Teoría económica institucional y creación de empresas. Investigaciones Europeas de Dirección y Economía de la Empresa, 11(3), 209-230.

Diario Oficial de Extremadura - DOE. (2007). Consejería de Educación - DOE $N^{\circ} 64$ - Orden de 24 de mayo. Recuperado de: http://doe.gobex.es/pdfs/ doe/2007/640o/640o.pdf 
Diario Oficial de Extremadura - DOE. (2011). Consejería de Educación - DOE $N^{\circ} 113$ - Orden de 31 de mayo - Red Extremeña de Escuelas Emprendedoras. Recuperado de http://culturaemprendedora.extremaduraempresarial.es/wpcontent/uploads/2012/09/Orden-31-de-Mayo-de-20111.pdf

Diario Oficial de Extremadura - DOE. (2012). Consejería de Educación y Cultura DOE $N^{\circ} 120$ - Decreto 108/2012, de 15 de junio, por el que se modifica el Decreto 82/2007, de 24 de abril, que establece el currículo de Educación Primaria para la Comunidad Autónoma de Extremadura. Recuperado de http://doe.gobex.es/pdfs/ doe/2012/1200o/12040119.pdf

Diario Oficial de Extremadura - DOE. (2012a). Consejería de Educación y Cultura - DOE $N^{\circ} 120$ - Decreto 109/2012, de 15 de junio, por el que se modifica el Decreto 83/2007, de 24 de abril, por el que se establece el currículo de Educación Secundaria Obligatoria para la Comunidad Autónoma de Extremadura. Recuperado de http://doe.gobex.es/pdfs/doe/2012/1200o/12040120.pdf

Fernández, A., Peña, I., Guerrero, M., y González, J.L. (2014). Global Entrepreneurship Monitor - Informe GEM España 2014. Recuperado de http://www.gem-spain. com/wp-content/uploads/2015/04/GEM_es_2014.pdf

Global Entrepreneurship Monitor - GEM Consortium. (2016). What is the national expert survey (NES). Recuperado de http://www.gemconsortium.org/wiki/1142

Global Entrepreneurship Monitor - GEM Consortium. (2016a). Country profile: Spain. Recuperado de http://www.gemconsortium.org/country-profile/109.

Gobierno de Extremadura. (2013). Programa de Desarrollo Rural de Extremadura FEADER 2007-2013. (Vol. I). Recuperado de http://www.gobex.es/filescms/ ddgg002/uploaded_files/fondos_europeos/PDR_Volumen_I.pdf

Gobierno de Extremadura.(2013a).Plan de Acción Integral de Empleo, Emprendedores y Empresa o Plan 3E. Recuperado de http://www.extremadura.ccoo.es/comunes/ recursos/10/doc80710_Plan_Empleo,_Emprendores_y_Empresa.pdf

Gobierno de Extremadura. (2014). Programa para el emprendimiento y la innovación social en Extremadura. Recuperado de http://www. emprendimientosocialextremadura.org/?page_id $=30$

Hernández, R., y Díaz, J.C. (2005). Global Entrepreneurship Monitor - Informe Ejecutivo 2005 - Extremadura. Recuperado de http://www.gem-spain.com/wpcontent/uploads/2015/03/GEM-Informe-Ext-2016.pdf

Hernández, R., Díaz, J.C., Sánchez, M.C., Fernández, A., Díaz, A.M., Almodóvar, M., y Rodríguez, R. (2013). Global Entrepreneurship Monitor - Informe Ejecutivo 2013. Extremadura. Recuperado de https://www.researchgate. net/publication/301550700_Global_Entrepreneurship_Monitor_Informe Ejecutivo_201415_EUROACE 
Hernández, R., Díaz, J.C., Sánchez, M.C., Fernández, A., Díaz, A.M., Almodóvar, M., Rodríguez, R., y Fernández, Y. (2014). Global Entrepreneurship Monitor Informe Ejecutivo 2014. Extremadura. Recuperado de https://www.researchgate. net/publication/301550700_Global_Entrepreneurship_Monitor_Informe Ejecutivo_201415_EUROACE

Hernández, C., y González, D. (2015, octubre). Estudio del ecosistema de emprendimiento tecnológico en Lima: estudio colectivo de casos. En O. Castellanos (Presidencia). XVI Congreso Latino-Iberoamericano de Gestión Tecnológica - ALTEC. Conferencia llevada a cabo en el XVI Congreso LatinoIberoamericano de Gestión Tecnológica - ALTEC 2015, Porto Alegre, Brasil.

Instituto de Estadística de Extremadura - IEEX. (2016). Demografía - Población - Datos Padrón Extremadura 2015. Recuperado de http://estadistica.gobex.es/ listadon2.php?id=9.

Instituto Nacional de Estadística - INE. (2016). Anuario Estadístico de España 2015-8. Mercado Laboral. Recuperado de http://www.ine.es/prodyser/pubweb/ anuario16/anu16_08merca.pdf.

Junta de Extremadura. (2008). Plan de Fomento y Calidad del Empleo en Extremadura 2008 - 2011. Recuperado de http://www.extremadura.ccoo.es/ comunes/recursos/10/doc7563_Plan_de_Fomento_y_Calidad_del_Empleo_en_ Extremadura.pdf.

Junta de Extremadura. (2010). Extremadura Avante, S.L.U. - Empresa pública. Recuperado de https://es.wikipedia.org/wiki/Extremadura_Avante

Junta de Extremadura. (2010a). Programa "Es tu oportunidad mujer emprendedora". Recuperado de http://sexpe.interface.es/matriculas/ programasEspecialidades/2127-es $\% 20$ tu $\% 20$ oportunidad $\% 20$ mujer $\% 20$ emprendedora.pdf

Junta de Extremadura. (2011). Consejo Extremeño de participación de las mujeres. Recuperado de https:/ciudadano.gobex.es/web/igualdad/consejo-extremeno-departicipacion-de-las-mujeres.

Junta de Extremadura. (2013, abril 17). La estrategia de emprendimiento femenino "Ser Empresaria" cuenta con una línea de un millón de euros en microcréditos. Noticias Junta de Extremadura. Recuperado de http://www.gobex.es/ comunicacion/noticia?idPub=8097\#.VyYwFPmLTIV.

Junta de Extremadura. (2015). Spain - Rural Development Programme (Regional) Extremadura 2014-2020. Recuperado de http://www.gobex.es/filescms/ddgg002/ uploaded_files/fondos_europeos/FondosEuropeos2014_2020/FEADER/ Programa_Desarrollo_Rural_2014.pdf 
Junta de Extremadura. (2016). Cultura emprendedora - Marco legal vigente. Recuperado de http://culturaemprendedora.extremaduraempresarial.es/?page id $=236$

Ministerio de Industria, Turismo y Comercio - Observatorio PYME. (2010). Servicios de apoyo a la persona emprendedora en España. Recuperado de http://www. ipyme.org/Publicaciones/EstudioServiciosApooyoEmprendedores.pdf

Poblete, C., y Amorós, J. E. (2013). University support in the development of regional entrepreneurial activity: an exploratory study from Chile. Investigaciones regionales, 26, 159-177.

Porter, M.E. (1991). La ventaja competitiva de las naciones. Barcelona: Plaza \& Janés Editores.

Reynolds, P., Hay, M., y Camp, R.M. (2002). Global Entrepreneurship Monitor 2002 Executive Report. Babson College, Kauffman Center for Entrepreneurial Leadership School Business (Eds.). London.

Sánchez, M.C., Postigo, M.V., Díaz, J.C., y Hernández, R. (2012). Análisis del estado del arte en el proyecto GEM sobre género y actividad emprendedora período 1999-2009. Economía Industrial, 383, 31-42.

Stel, A., Carree, M., y Thurik, R. (2005). The effect of entrepreneurial activity on national economic growth. Small Business Economics, 24, 311-321.

Stevenson, L. (2002). Politicas de desarrollo empresarial en el mundo. XV Congreso Latinoamericano sobre Espíritu Empresarial - Las Pymes como alternativa de desarrollo para América Latina. Bogotá, Colombia.

Universidad de Extremadura - UEX. (2016). Presentación de la UEX. Recuperado de http://www.unex.es/conoce-la-uex/presentacion

Urbano, D.; Rojas, A. y Díaz, J.C. (2010). ¿Hacia dónde va la investigación en el proyecto GEM? Revista Europea de Dirección y Economía de la Empresa, 19(2), $15-30$.

World Economic Forum - WEF. (2009). Educating the Next Wave of Entrepreneurs. Recuperado de http://www3.weforum.org/docs/WEF_GEI_ EducatingNextEntrepreneurs_ExecutiveSummary_2009.pdf 